\title{
Treatment-Induced Acute Leukaemia after Major Response to Cyclophosphamide-Based Metronomic Chemotherapy in Refractory Heavily Pre-Treated Prostate Cancer
}

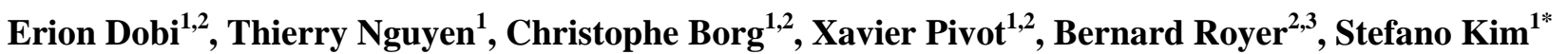 \\ ${ }^{1}$ Department of Medical Oncology, University Hospital of Besançon, Besançon, France; ${ }^{2}$ Institut National de la Santé et de la Re- \\ cherche Médicale-Unité Mixte de Recherche (INSERM UMR) No. 1098, Besançon, France; ${ }^{3}$ Department of Pharmacology, Univer- \\ sity Hospital of Besançon, Besançon, France. \\ Email: * stefanokim@gmail.com
}

Received November $12^{\text {th }}, 2012$; revised December $13^{\text {th }}, 2012$; accepted December $21^{\text {st }}, 2012$

\begin{abstract}
Background: Metronomic chemotherapy is based on antiangiogenic and immunologic mechanisms obtained by the administration of traditional cytotoxic drugs at lower concentration without rest periods. The low dosage induces fewer or no side effect compared to classic maximum tolerated dose administration (MTD). At present, no treatment related acute leukaemia was reported in cyclophosphamide-based metronomic chemotherapy (CMC). Case: We report the case of an 81-year-old man considered as having castration and chemo-refractory metastatic prostate cancer. CMC was started. Objective response was observed in this heavily pre-treated patient with progression free survival lasting more than 30 months. No toxicity was observed in this period and his autonomy was maintained. Finally, our patient developed a chemotherapy-induced acute myeloid leukaemia at 36th month of CMC. Conclusion: Even CMC is a well-tolerated treatment; secondary acute leukaemia is related to cumulative dose of cyclophosphamide. The benefit and the risk of long-term exposure to cyclophosphamide should be carefully balanced.
\end{abstract}

Keywords: Metronomic Chemotherapy; Cyclophosphamide; Prostate Cancer; Secondary Acute Leukemia

\section{Introduction}

Classic Maximum Tolerated Dose administration (MTD) is by definition the highest dose of a drug or treatment that does not cause unacceptable side effects. Most of chemotherapy protocols were developed based in this concept [1].

Metronomic chemotherapy is the administration of traditional cytotoxic drugs at lower concentration without rest periods. The basis of this particular modality is supported by its antiangiogenic effect [2-5]. Moreover, cyclophosphamide-based metronomic chemotherapy (CMC) enhances immune response against tumour by inhibition of $\mathrm{CD}^{+} 25^{+} \mathrm{T}$ regulatory cell function [6], whereas higher doses of cyclophosphamide are associated with cytotoxicity and immunosuppression $[7,8]$.

Several phase II trial analyzed the interest of CMC in different tumours, only or in combination [9-15]. Most interesting results were observed in breast and prostate cancer. In monotherapy, tolerability is excellent and

"Corresponding author. grade III-IV non-haematological toxicities are virtually absent [12-14]. At present, no treatment related acute leukaemia was reported with CMC.

We report here the first case of treatment related acute myeloid leukaemia secondary to long exposure to CMC.

\section{Case Report}

A 77-year-old man was referred to our institution for refractory metastatic synchronous bladder and prostate carcinoma. Three years before, a urinary obstruction conducted a diagnosis of a high-grade prostate adenocarcinoma extending through the bladder muscle (stage pT4, Gleason score 8). PSA (Prostate Serum Antigen) at diagnosis was $3.4 \mathrm{ng} / \mathrm{ml}$. CT scan, bone scintigraphy, and MRI (Magnetic Resonance Imaging) showed osteoblastic metastases at his right hipbone. A medical castration by androgen deprivation therapy was started without locoregional treatment. Two years before, a cystoscopic examination showed an invasive transitional-cell carcinoma localized in urethra extended into prostate tissue (stage pT4a). Cystoprostatectomy was rejected in this 
patient with metastatic prostatic cancer. One year before, he presented a pain in pelvic region and PSA raised to 15 $\mathrm{ng} / \mathrm{ml}$. New endoscopic examination showed a large infiltration of the bladder by transitional-cell carcinoma and persistence of Gleason 8 adenocarcinoma at prostate resection. Bone scintigraphy and CT scan documented multiple pulmonary metastases and progression of bone metastases. An oral anti-androgen treatment and zoledronic acid were added to LHRH (Luteinizing HormoneReleasing Hormone) analogue. Radiotherapy of pelvis was undertaken and then, a mono-chemotherapy by gemcitabine was started.

The patient was a retired military nurse, single, and lived half of the time in Equator. There was no history of alcohol or tobacco intoxication, and he was otherwise in good health and the rest of patient's medical history was unremarkable.

At admission, his only complaint was his intermittent pain in his hip. CT scan showed stable disease, but PSA increased to $14 \mathrm{ng} / \mathrm{ml}$. Oral anti-androgen was replaced by estramustine, and gemcitabine was maintained. Seven months later, the PSA rose to $67 \mathrm{ng} / \mathrm{ml}$. CT scan and bone scintigraphy disclosed pulmonary and bone progression. He presented grade 1 asthenia. Several lines of chemotherapy were prescribed: Carboplatin-paclitaxel for 6 months, Phase I trial with vinflunine for 2 months, and paclitaxel for 4 months. Important limiting toxicities were observed at each protocol. The disease progressed soon after its stop, and clinical deterioration became evident (ECOG Performance Status at 2). In total, in 30 months, 4 lines of chemotherapy were tempted.

A new treatment by $\mathrm{CMC}$ at dose of $50 \mathrm{mg}$ a day without interruption was proposed. Six months later, new CT scan showed a surprising partial response (Figure 1). PSA decreased to $19.9 \mathrm{ng} / \mathrm{ml}$. The tolerability was excellent. We encouraged the patient to continue $\mathrm{CMC}$ regimen. One year later a new CT scan revealed a major pulmonary response and PSA decreased to $6.6 \mathrm{ng} / \mathrm{ml}$ (Figure 2). This response lasted during 30 months until PSA rose to $31 \mathrm{ng} / \mathrm{ml}$ but the CT scan showed no evidence of pulmonary progression (Figure 3). New hormone therapy by diethylstilbestrol was started. Unfortunately, six months later, an acute myeloid leukaemia (AML) was diagnosed. Cytogenetic study confirmed a treatment-induced leukaemia with loss of whole chromosome 5, partial monosomie of chromosome 17 and 18 $(43, \mathrm{X},-\mathrm{Y},-5, \mathrm{t}(17 ; 18),-18)$. He decided to return to South America to continue palliative transfusion therapy.

\section{Discussion}

Metronomic chemotherapy is based on dual antiangiogenic and immunologic mechanisms obtained by the administration of traditional cytotoxic drugs given con-
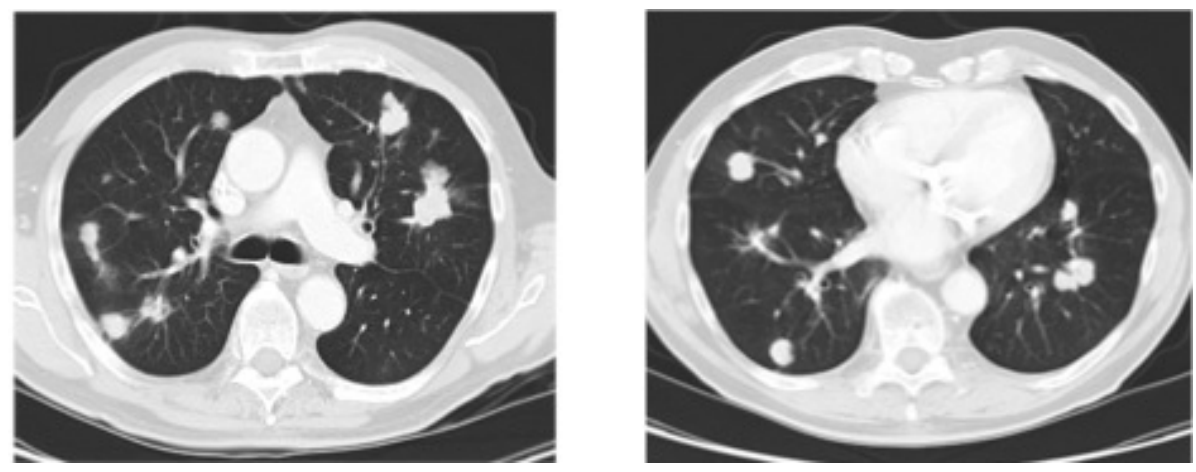

Figure 1. 6 months after cyclophosphamide start.
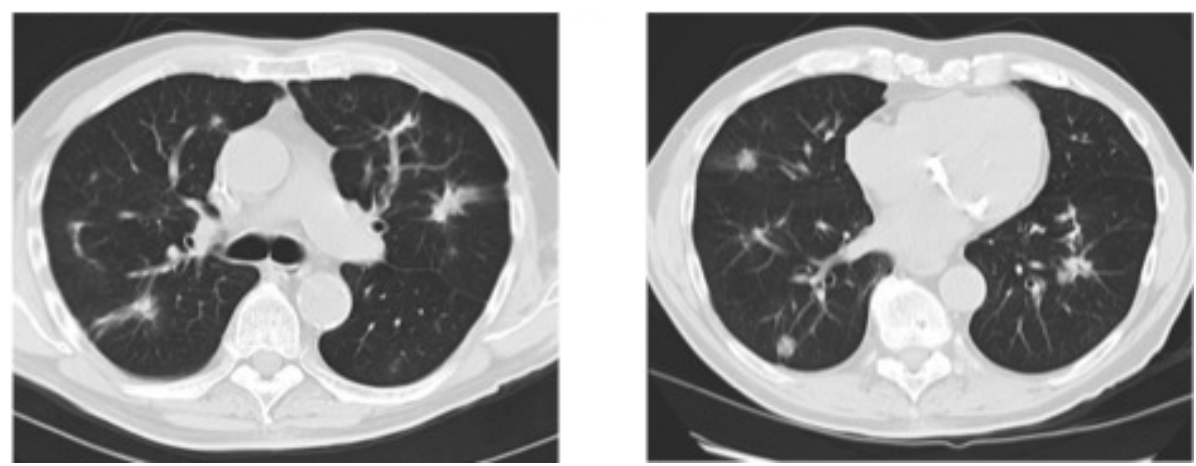

Figure 2. 18 months after cyclophosphamide start. 

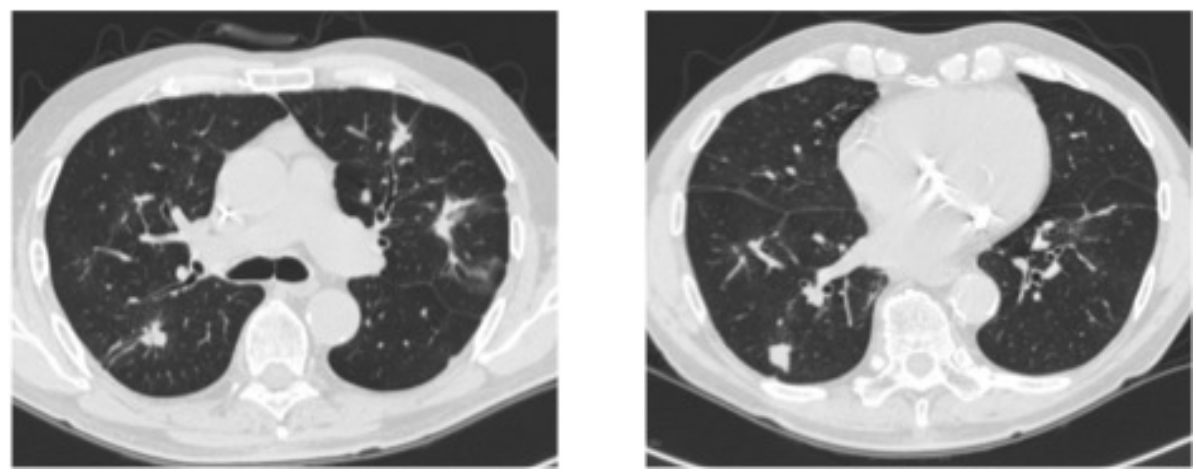

Figure 3. 28 months after cyclophosphamide start.

tinuously and at low concentration. Low doses of cyclophosphamide are able to enhance immune response against a variety of antigens. In murine model, metronomic cyclophosphamide has shown a decrease activity of regulatory $\mathrm{T}$ cells $\left(\mathrm{T}_{\mathrm{REGs}}\right)$ implicated in tolerance to self-antigens, such as tumour-associated antigens [6]. Moreover, endothelial cells required in the neo-angiogenic process suffer from the chemotherapy toxic effect as any other dividing cells. It is well established that under metronomic regimen endothelial cell sub-lethal or lethal alterations are observed and the continuous exposure may definitively compromise their recovery [2-5].

Maximum Tolerated Dose (MTD) regimen was and still is the main way to manipulate chemotherapy drugs to treat solid tumours in view of dose-intensity effect. However, this does not take in consideration the interacttion between tumour cells and its complex environment such as stroma, vessels, growth factors, and immune system among others. Besides, the main gold of MTD is to kill tumour cells and this regimen is especially valid when tumour shrinkage is required with potential curative-intent surgery. In palliative setting, the clinical endpoint is overall survival or quality-of-life. Tumour response rate by RECIST criteria is not a good surrogate end-point for survival benefice $[16,17]$. Recently, a phase III trial in advanced melanoma treated with ipilimumab, an immunotherapy, showed that the survival benefice is not even related to tumour progression [18].

$\mathrm{CMC}$ is a complete different way to treat cancer cells than classic MTD regimen since its main mechanism is the inhibition of angiogenesis and enhancement of immune response decreasing $\mathrm{T}_{\mathrm{REGs}}$ implicated in tolerance of tumour cells. Consequently, CMC's target is the tumour environment instead of tumour cell itself.

CMC regimen was already used in prospective trials in other cancers. In breast cancer, CMC in combination with low dose methotrexate (MTX) was used in a phase II trial as second line treatment in metastatic setting. The disease control rate was $31.7 \%$, comparable to other second line regimens. Twenty-six per cent of patients were still responding after 12 months [10]. Cost/effective analysis also favoured this regimen [11].

In prostate cancer, two prospective phase II study evaluated $\mathrm{CMC}$ regimen as monotherapy in castration refractory prostate cancer (CRPC). Raghavan et al. evaluated 30 patients with measurable disease. Disease control was observed in 18 of 30 patients (6 PR, and $12 \mathrm{SD}$ ), and median OS was 12.7 months. Lord et al. evaluated 80 CRPC patients. Overall response rate was $34.5 \%$, and the PFS was 7.5 months. The treatment was well tolerated. The only grade III-IV toxicities were haematological, mostly lymphopenia [12,13]. Treatment efficacy was also observed in different combination regimens with CMC [14].

In transitional bladder cancer, a phase I/II trial with paclitaxel at classic MTD of $175 \mathrm{mg} / \mathrm{m}^{2}$ and cyclophosphamide $50 \mathrm{mg}$ day 1 to 7 was undertaken. In this group, partial response rate was $31 \%$, time to progression and median OS were 5 and 8 months, respectively [15].

In our case, the patient had a castration-refractory bone metastatic prostate cancer and a muscle and prostate invasive transitional bladder cancer. Unfortunately, pulmonary metastases have never been biopsied. However in retrospective analysis, its history was more likely from prostate origin than transitional bladder cancer. PSA concurred with lung metastases evolution and the long control of advanced disease by CMC in monotherapy was clearly described in the literature for prostate cancer but not for bladder origin. Four different regimens of chemotherapy were used before CMC. He was not considered refractory to chemotherapy since stable disease was noted after each regimen but no objective response was seen and disease progression was observed soon after each stop. Toxicity was sometimes important and a dose limiting factor. CMC was prescribed as fifth line chemotherapy. The treatment was well tolerated and pursued without interruption. Surprisingly, objective partial response was documented and PFS was 30 months (equivalent to all four precedent treatments together in earlier stage). Biologic progression was finally docu- 
mented without radiologic progression until his acute leukaemia was diagnosed (after 36 months of treatment by $\mathrm{CMC}$ ).

AML can be secondary cancer related to chemotherapy. Even though there is no publication associating AML to metronomic cyclophosphamide, is highly possible. In the trial of Colleoni et al. in breast cancer, cyclophosphamide was continued up to 2 years but no second cancer was described. In prostate cancer, no case of leukaemia was reported. In a prospective phase III trial of rhabdomyosarcoma treated with high dose of cyclophosphamide, the incidence density of developing acute myeloid leukaemia was 7.6, in comparison to 51.6 when cyclophosphamide and etoposide were given and 0 when patients did not received neither cyclophosphamide nor etoposide. The median time to development of AML in these cases was 39 months [19]. In a cohort of 3412 patients treated for non-Hodgkin lymphoma including 118 patients with secondary malignancy, a higher risk of leukemia as restricted to patients given cumulative dose of cyclophosphamide more than $11,250 \mathrm{mg} / \mathrm{m}^{2}$ [20,21]. Our patient received $50 \mathrm{mg}$ daily dose without interrupttion for 36 months with consequent cumulative dose of more than $60,000 \mathrm{mg}$. However he was also exposed to other leukemic potential drugs and their contribution to development of secondary AML cannot be excluded. The three unbalanced abnormalities observed in our patient (complete monosomie of chromosome 5 and partial monosomie of chromosome 17 and 18 are most frequently observed in alkylant-induced AML [19].

\section{Conclusions}

$\mathrm{CMC}$ is a well-tolerated treatment and clinical benefit was evident in our patient. This excellent tolerance profile and efficacy led us to pursuit the treatment for 36 months with consequent cumulative total dose of more than $60 \mathrm{~g}$ of cyclophosphamide. Even though treatmentrelated leukaemia with $\mathrm{CMC}$ was not published, acute leukaemia related to cumulative dose of cyclophosphamide is well described in the literature. However, clinical oncologists rarely bare in mind this potential risk of cyclophosphamide-based protocols and can be particularly dangerous in case of CMC with good tumour control since clinical or biological limiting toxicities are extremely rare.

Treatment-related acute leukaemia should be considered in case of CMC treatment, and the benefit and the risk of long-term exposure to cyclophosphamide should be carefully balanced.

\section{REFERENCES}

[1] http://www.cancer.gov/dictionary?cdrid=546597
[2] R. S. Kerbel and B. A. Kamen, "The Anti-Angiogenic Basis of Metronomic Chemotherapy," Nature, Vol. 4, No. 6, 2004, pp. 423-436.

[3] D. Hanahan, G. Bergers and E. Bergsland, "Less is More, Regularly: Metronomic Dosing of Cytotoxic Drugs Can Target Tumor Angiogenesis in Mice," Journal of Clinical Investigation, Vol. 105, No. 8, 2000, pp. 1045-1047. doi:10.1172/JCI9872

[4] S. Man, G. Bocci, G. Francia, S. K. Green, S. Jothy, D. Hanahan, et al., "Antitumor Effects in Mice of Low-Dose (Metronomic) Cyclophosphamide Administered Continuously through the Drinking Water," Cancer Research, Vol. 62, No. 10, 2002, pp. 2731-2735.

[5] Y. Hamano, H. Sugimoto, M. A. Soubasakos, M. Kieran, B. R. Olsen, J. Lawler, et al., "Thrombospondin-1 Associated with Tumor Microenvironment Contributes to Low-Dose Cyclophosphamide-Mediated Endothelial Cell Apoptosis and Tumor Growth Suppression," Cancer Research, Vol. 64, No. 5, 2004, p. 1570. doi:10.1158/0008-5472.CAN-03-3126

[6] J. E. Damber, C. Vallbo, P. Albertsson, B. Lennernas and K. Norrby, "The Anti-Tumour Effect of Low-Dose Continuous Chemotherapy May Partly Be Mediated by Thrombospondin," Cancer Chemotherapy and Pharmacology, Vol. 58, No. 3, 2006, pp. 354-360. doi:10.1007/s00280-005-0163-8

[7] M. E. Lutsiak, R. T. Semnani, R. De Pascalis, S. V. Kashmiri, J. Schlom and H. Sabzevari, "Inhibition of CD4(_25_T Regulatory Cell Function Implicated in Enhanced Immune Response by Low-Dose Cyclophosphamide," Blood, Vol. 105, No. 7, 2005, pp. 2862-2868. doi:10.1182/blood-2004-06-2410

[8] J. Mayer, M. Krejcí, M. Doubek, Z. Pospísil, Y. Brychtová, et al., "Pulse Cyclophosphamide for CorticoidRefractory Graft-Versus-Host Disease," Bone Marrow Transplantation, Vol. 35, No. 7, 2005, pp. 699-705. doi:10.1038/sj.bmt.1704829

[9] A. Reiner, T. Gernsheimer and S. J. Slichter, "Pulse Cyclophosphamide Therapy for Refractory Autoimmune Thrombocytopenic Purpura," Blood, Vol. 85, No. 2, 1995, pp. 351-358.

[10] N. Penel, A. Adenis and G. Bocci, "CyclophosphamideBased Metronomic Chemotherapy: After 10 Years of Experience, Where Do We Stand and Where Are We Going?" Critical Reviews in Oncology/Hematology, Vol. 82, No. 1, 2011, pp. 40-50.

[11] M. Colleoni, A. Rocca, M. T. Sandri, et al., "Low-Dose Oral Methotrexate and Cyclophosphamide in Metastatic Breast Cancer: Antitumor Activity and Correlation with Vascular Endothelial Growth Factor Levels," Annals of Oncology, Vol. 13, No. 1, 2002, pp. 73-80. doi:10.1093/annonc/mdf013

[12] G. Bocci, M. Tuccori, U. Emmenegger, V. Liguori, A. Falcone, R. S. Kerbel and M. Del Tacca, "Cyclophosphamide-Methotrexate 'Metronomic' Chemotherapy for the Palliative Treatment of Metastatic Breast Cancer. A Comparative Pharmacoeconomic Evaluation," Annals of Oncology, Vol. 16, No. 8, 2005, pp. 1243-1252. 
doi:10.1093/annonc/mdi240

[13] D. Raghavan, K. Cox, B. S. Pearson, et al., "Oral Cyclophosphamide for the Management of Hormone-Refractory Prostate Cancer," British Journal of Urology, Vol. 72, No. 5, 1993, pp. 625-628.

doi:10.1111/j.1464-410X.1993.tb16222.x

[14] R. Lord, S. Nair, A. Schache, J. Spicer, N. Somaihah, V. Khoo and H. Pandha, "Low Dose Metronomic Oral Cyclophosphamide for Hormone Resistant Prostate Cancer: A Phase II Study," Journal of Urology, Vol. 177, No. 6, 2007, pp. 2136-2140. doi:10.1016/j.juro.2007.01.143

[15] T. Nelius, K. Rinard and S. Filleur, "Oral/Metronomic Cyclophosphamide-Based Chemotherapy as Option for Patients with Castration-Refractory Prostate Cancer: Review of the Literature," Cancer Treatment Reviews, Vol. 37, No. 6, 2011, pp. 444-455. doi:10.1016/j.ctrv.2010.12.006

[16] G. Di Lorenzo, V. Montesarchio, R. Autorino, T. Bellelli, N. Longo, et al., "Phase 1/2 Study of Intravenous Paclitaxel and Oral Cyclophosphamide in Pretreated Metastatic Urothelial Bladder Cancer Patients," Cancer, Vol. 115, No. 3, 2009, pp. 517-523. doi:10.1002/cncr.24055

[17] T. R. Fleming, "Objective Response Rate as a Surrogate End Point: A Commentary," Journal of Clinical Oncolo- gy, Vol. 23, No. 22, 2005, pp. 4845-4846. doi:10.1200/JCO.2005.92.008

[18] T. R. Fleming, "Surrogate End Points and FDA's Accelerated Approval Process," Health Affairs, Vol. 24, No. 1, 2005, pp. 67-78. doi:10.1377/hlthaff.24.1.67

[19] F. S. Hodi, S. J. O’Day, D. F. McDermott, R. W. Weber, J. A. Sosman, et al., "Improved Survival with Ipilimumab in Patients with Metastatic Melanoma," The New England Journal of Medicine, Vol. 363, No. 8, 2010, pp. 711-723. doi:10.1056/NEJMoa1003466

[20] N. Mauritzson, M. Albin, L. Rylander, R. Billström, T. Ahlgren, et al., "Pooled Analysis of Clinical and Cytogenetic Features in Treatment-Related and de Novo Adult Acute Myeloid Leukemia and Myelodysplastic Syndrome Based on a Consecutive Series of 761 Patients Analyzed 1876-1993 and on 5098 Unselected Cases Reported in the Literature 1974-2001," Leukemia, Vol. 16, No. 12, 2002, pp. 2366-2378. doi:10.1038/sj.leu.2402713

[21] Y. Xu, H. Wang, S. Zhou, M. Yu, X. Wang, et al., "Risk of Second Malignant Neoplasms after Cyclophosphamide-Based Chemotherapy with or without Radiotherapy for Non-Hodgkin's Lymphoma," Leukemia \& Lymphoma, 26 November 2012, [Epub ahead of print] . doi:10.3109/10428194.2012.743657 